Chemdata or the Imperial Chemical Industries' Information Register for information on health hazards, fire fighting techniques, and clean up procedures for spills. All staff should ensure that they do not put themselves at risk, and when appropriate the fire brigade will decontaminate casualties before they receive other than immediate lifesaving treatment.

Health professionals are more used to disaster planning for major trauma than for mass chemical exposures and the medical management problems these pose. ${ }^{5}$ As a result the level of preparedness of accident and emergency departments in the United Kingdom is variable with regard to training, the use of chemical incident protocols, the provision of protective clothing and antidotes, and the availability of facilities for decontaminating seriously ill casualties. ${ }^{2}$ In this issue Thanabalasingham et al illustrate such deficiencies in an actual incident and highlight the risks that emergency teams may face as a consequence ( $\mathrm{p} 101) .{ }^{13}$

There is always the risk that the medical response to the uncommon but major incident might be delayed or mismanaged because the epidemiological, laboratory, and toxicological skills needed rapidly to evaluate and advise on the hazard are not available locally. ${ }^{5}$ A team of experts may need to be deployed to make an urgent clinical and epidemiological assessment of the health impact and to ascertain the sources and extent of a toxic exposure in the population. This also applies to outbreaks of toxic illness from ingesting contaminated food or water, which might first come to the attention of general practitioners or specialists in public health medicine $\mathrm{e}^{1+}$; a recent example was the Lowermoor incident in 1988, when water was contaminated with aluminium sulphate. Until now there has been no provision for deploying such back up for local emergency services in the United Kingdom and no central government responsibility for coordinating major chemical incidents in peacetime, ${ }^{15}$ but the Lowermoor incident has prompted the government to take some action. The Department of Health has recently formed an independent health advisory group to provide advice to health officials - urgently and on request-confronted with serious chemical contamination of a water supply. Local and national medical planning for chemical incidents needed strengthening. The many agencies and professional groups concerned need to develop much closer links and identify nationally the sources of expertise available in an emergency.

Consultant Occupational Physician,

PETER J BAXTER

University Department of Community Medicine,

Addenbrooke's Hospital,

Cambridge CB2 2QQ

1 Medvedev Z. The legacy of Chernobyl. London: Blackwell Scientific, 1990

2 Murray V, ed. Major chemical disasters: medical aspects of management. London: Royal Society of Medicine, 1990.

3 Canadine IC. The possibility of major incidents in chemical distribution. In: Murray V, ed. Major chemical disasters: medical aspects of management. London: Royal Society of Medicine, 1990:33-9.

+ Bertazzi PA, Zocchetti C, Pesatori AC, Guercilena S, Sanarico M, Radice L. Ten-year mortality of the population involved in the Seveso incident in 1976. Am I E pidemiol 1989;129:1187-200.

5 Baxter PJ. Review of major chemical accidents and their medical management. In: Murray V, ed. Major chemical disasters: medical aspects of management. London: Royal Society of Medicine. 1990:7-20.

6 World Health Organisation. Toxic oil syndrome. Copenhagen: WHO, 1984

Health and Safety Executive. The control of major industrial accident hazards regulations 1984 (CIMAH): further guidance on emergency plans. London: HMSO, 1986.

8 Baxter PJ, Davies PC, Murray V. Medical planning for toxic releases into the community: the example of chlorine gas. Br $\mathrm{F}$ Ind Med 1989;46:277-85.

9 Health and Safety Executive. Risk criteria for land use planning in the vicinity of major industrial hazards. London: HMSO, 1989.

10 Purdy G, Davies PC. Toxic gas incidents - some important considerations for emergency planning. In: The assessment and control of major hazards. Rugby: Institute of Chemical Engineers, 1985:367-88.

11 Binder S. Deaths, injuries, and evacuations from acute hazardous materials releases. Am f Public Health 1989;79:1042-3.

12 Duclos P, Binder S, Riester R. Community evacuation following the Spencer metal processing plant fire, Nanticoke, Pennsylvania. Journal of Hazardous Materials 1989;22:1-11.

13 Thanabalasingham T, Beckett MW, Murray V. Report of a chemical incident from ethyldichlorosilane. BMF 1991;302:101-2.

14 Volans GN. Medical management of chemical disasters involving food or water. In: Murray V, ed. Major chemical disasters: medical aspects of management. London: Royal Society of Medicine, 1990:173-9.

15 Stealey J. Planned response: civil defence. In: Murray V, ed. Major chemical disasters: medical aspects of management. London: Royal Society of Medicine, 1990:103-8.

\title{
Transient global amnesia
}

\section{Recurrences are rare and patients may drive}

The syndrome of transient global amnesia first became clearly recognised with the description of 17 cases by Fisher and Adams in $1964 .^{\prime}$ Some two years later the late Lord Brain described a series of cases at a meeting at the Association of British Neurologists, and it was clear from the discussion that most neurologists had seen examples of the condition. Transient global amnesia is now a well established clinical entity with extensive reports describing more than 1000 cases. $^{2}$

Transient global amnesia typically occurs in a middle aged or elderly person, who suddenly develops a disorder of memory, often regarded as confusion, which lasts for some hours. During this time the registration and recall of current events are impaired and afterwards the victim cannot remember any of the events during the confused period. During the attack the patient seems healthy, though is often distressed and not aware of what is wrong. There is no loss of personal identity and complex functions such as driving may be performed without difficulty. Recovery is complete and recurrences are unusual.

The pattern of memory disturbance has been the subject of much interest for several years, though neuropsychological function during attacks has rarely been examined. Hodges and Ward managed to examine five patients during an attack and were able to show a characteristic neuropsychological deficit. ${ }^{3}$ Personality, problem solving, language, and visuospatial function remained intact. Immediate memory was preserved, but longer term verbal and non-verbal memory was severely disrupted. Retrograde amnesia was commonly present, though this tended to diminish during recovery, leaving a short retrograde gap in all cases. These observations confirm that transient global amnesia is primarily a disorder of memory and are compatible with the widely held view that the cause of the trouble lies within the temporal lobes.

Aetiologically transient global amnesia remains an enigma, though the most widely accepted view is that it is due to thromboembolic cerebrovascular disease with ischaemia in the territory of the posterior cerebral arteries, which supply the medial temporal lobes $^{4}$-in other words, a transient ischaemic attack within the vertebrobasilar system. Other views are that the syndrome is caused by epilepsys or migraine. ${ }^{6}$ Evidence against the transient ischaemic attack theory is that the attacks last longer than an ordinary transient ischaemic attack, repeated attacks are rare, and strokes within the appropriate vascular territory, producing permanent memory loss, are exceptional. ${ }^{3}$

A new study has provided some important information about this disorder and its possible cause. Hodges and 
Warlow performed a case-control comparison of 114 patients with transient global amnesia, 109 normal community based controls, and 212 patients with transient ischaemic attacks. ${ }^{2}$ The results of this careful study do not support a cerebrovascular cause for transient global amnesia. Firstly, there were no significant differences in the prevalence of vascular risk factors between the patients with transient global amnesia and normal controls; secondly, those with transient global amnesia and those with transient ischaemic attack were not matched for vascular risk factors; and, thirdly, during follow up patients subject to transient ischaemic attacks had a much greater incidence of vascular events and a higher mortality than those subject to transient global amnesia. On the other hand, migraine was more common in those with transient global amnesia than in those in both control groups, though there were no differences in the prevalence of epilepsy.

Unfortunately this information does not enable us to reach a firm conclusion about the cause of transient global amnesia, which in most cases remains uncertain. Thromboembolic disease may be the cause in a few cases, and migraine and epilepsy might account for some more. At a practical level no treatment will reduce the risk of recurrence, though the condition is benign and in general the patient can be reassured. Up to now, believing the transient ischaemic attack theory, my own advice to patients has been that they should not drive for six months after an episode of amnesia. Based on the recent evidence, however, the licensing authority in Swansea now recommends no restrictions to holders of an ordinary driving licence and a one year restriction to holders of a vocational licence.

Senior Lecturer in Neurology,

N E F CARTLIDGE

School of Neurosciences,

University of Newcastle upon Tyne,

Newcastle upon Tyne NE2 4HH

1 Fisher CM, Adams RD. Transient global amnesia. Acta Neurol Scand 1964;40(suppl 9):1-83.

2 Hodges JR, Warlow CP. The aetiology of transient global amnesia. A case-control study of 114 cases with prospective follow up. Brain 1990;113:639-57.

3 Hodges JR, Ward CD. Observations during transient global amnesia. Brain 1989;112:595-620.

4 Kaplan LB. Transient global amnesia. In: Vinken PJ, Bruyn GW, Klawans HL, eds. Handbook of clinical neurology. vol 45. Amsterdam: Elsevier, 1985:205-18.

Fisher CM. Transient global amnesia; precipitating activities and other observations. Arch Neurol 1982;39:605-8.

6 Kaplan L, Chedru F, L'Hermitte F, Mayman C. Transient global amnesia and migraine. Neurology 1981;31:1169-70.

\title{
Surrogate end points in clinical trials
}

\author{
Getting closer to identifying markers for survival in AIDS
}

The ultimate goals of developing new treatments for a fatal disease are, in order of priority: cure, prolonging life, and reducing morbidity without adversely affecting survival. The data required to assess these goals are often obtainable only after a long period of patient follow up as we must observe a certain number of deaths to estimate the survival time reliably.

Unfortunately, the study of fatal diseases generates the greatest pressure to evaluate new drugs rapidly. The desire to reach conclusions about drugs without waiting for survival differences to be definitively established naturally leads to a search for other, more proximal events that might equally well (or even better) reflect therapeutic benefit. Examples of such surrogate end points in common use are blood pressure (for cardiovascular mortality) ${ }^{1}$ and tumour enlargement and relapse (for cancer mortality). ${ }^{2}$ Surrogate end points are also used in non-fatal chronic diseases of organ systems - for example, intraocular pressure is used as a surrogate for long term visual function. ${ }^{3}$ These end points are generally accepted as valid for assessing therapeutic efficacy and have served as the basis for regulatory agencies' approval of drugs.

Clinical trials in AIDS are already using surrogate end points. Recently completed trials of zidovudine in patients in the early stages of HIV infection used progression of disease rather than death as the primary end point. ${ }^{+5}$ In a continuing placebo controlled trial of zidovudine in asymptomatic patients with adequate numbers of CD4 + cells a decline to a count of $0.5 \times 10^{9} / 1$ or below has been defined as a primary end point because zidovudine has already been shown to be effective for patients at these lower counts. Antiviral drug trials in patients with more advanced disease, however, have generally remained dependent on mortality as the primary end point.

Certain biological markers have aroused intense interest as surrogates for survival time in evaluating new treatments for AIDS and HIV infection. The effect of a drug on these markers tends to be observable quickly, often within a few weeks after starting treatment. Several such markers have been shown to be strongly associated with the probability of long term survival, with the greatest attention given to the CD4+ count. ${ }^{6}$ Nevertheless, a correlation between a marker value and ultimate survival time does not necessarily imply that a treatment's effect on the marker will predict the treatment's effect on survival. ${ }^{7}$ For example, a treatment might stabilise or even improve the marker value while producing other adverse effects that tend to shorten survival. A recent example of just such a finding outside AIDS is instructive. Arrhythmias occurring after myocardial infarction are known to be associated with poorer survival; yet a clinical trial of effective antiarrhythmic drugs showed that patients who took these drugs were more likely to die than those who received placebo. ${ }^{8}$ Another concern is that effects on markers may be insufficiently sustained to produce a measurable improvement in clinical outcome.

How, then, can we assess the validity of a potential surrogate marker for trials of AIDS treatment? Certainly there should be a strong biological rationale, and it should also be evident that the marker value at a given point in time is strongly predictive of ultimate survival time. Both of these criteria are met by several markers. What is also required, and what has not been shown for any marker, is that the effect of treatment on the surrogate end point will reliably predict the effect of treatment on survival for individual patients. The paper by Jacobson et al (p 73) takes an important step in this direction. ${ }^{9}$ The authors have gone beyond simply showing the prognostic values of the markers and have investigated whether a change in marker values over time correlates with long term survival. In their analysis of 90 patients receiving zidovudine during trials only changes in $\beta_{2}$ microglobulin concentrations were associated with survival time after they had adjusted for selected baseline characteristics. Absolute CD4+ counts by weeks 8-12-but not changes from baseline counts-provided additional predictive value. p24 antigen concentrations, whether considered as changes from baseline 